\title{
Erfolgreich Geldauflagen einwerben
}

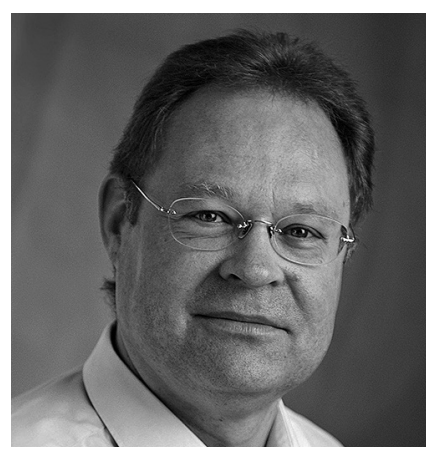

VON WILHELM HEERMANN Wilhelm Heermann ist Inhaber der pro fund Kommunikation \& Fundraising Management und pro fund Media Services GmbH. Er hat neun Jahre als Fundraiser in einer Entwicklungsorganisation gearbeitet und ist seit fünfzehn Jahren als selbstständiger Fundraiser für gemeinnützige Einrichtungen tätig. Neben dem GeldauflagenMarketing gehören die strategische Beratung, die Entwicklung und Umsetzung von Medienkampagnen, Unternehmenskooperationen und Personalrecruitment ins Portfolio von pro fund. www.pro-fund.de

\author{
Geldauflagen können für Verbände und soziale \\ Unternehmen eine zusätzliche Finanzierungsquelle \\ darstellen. Bis zu 150 Millionen Euro werden \\ in Deutschland jedes Jahr von Gerichten und \\ Staatsanwaltschaften gemeinnützigen Organisationen \\ zugesprochen. Allerdings gibt es beim Versuch, \\ Geldauflagen einzuwerben, einiges zu beachten.
}

In den letzten Jahren haben die Anfragen nach Geldauflagen - umgangssprachlich als »Bußgelder« bezeichnet - stark zugenommen, deshalb ist heute eine differenziertere Ansprache und eine gute Kenntnis über den "Zuweisermarkt" notwendig, um die eigene Organisation optimal positionieren zu können.

Dass Geldauflagen nicht nur der Staatskasse zufallen, sondern auch gemeinnützigen Einrichtungen $\mathrm{zu}-$ gesprochen werden können, stellt in Deutschland, im Vergleich zu unseren Nachbarländern, eine Besonderheit dar. Der Gesetzgeber hat aber mit der Möglichkeit, unter bestimmten Umständen auf ein Verfahren durch die Annahme der Zahlung einer Geldauflage verzichten zu können, bestimmt eher prozessökonomische Aspekte im Vordergrund gesehen.

In den letzten Jahren sind besonders Entscheidungen im Vorfeld der Ermittlung von Wirtschaftskriminalität bekannt geworden. So beispielsweise die Geldauflage gegen den früheren PostChef Zumwinkel, der 2009 neben einer zur Bewährung ausgesetzten Haftstrafe, eine Geldauflage von einer Million Euro gezahlt hat. Die Kritik über die Verteilung dieses Geldes durch die im Ermittlungsverfahren beteiligte Staatsanwältin Lichtinghagen an ihr nahestehende gemeinnützige Einrichtungen, füllten nicht nur tagelang die Presseberichte, sondern hat auch die Verteilpraxis geändert. Recht preiswert kam Heinrich von
Pierer davon, der während der Zeit, als Siemens Schmiergelder verteilte, Konzern- und später Aufsichtsratschef war. Bei 1,3 Milliarden Euro in schwarzen Kassen und 2,5 Milliarden Euro Gesamtschaden reichten die Beweise lediglich für eine Geldauflage von 250.000 Euro.

Neben diesen populären Beispielen werden in deutschen Amtsstuben in der Regel viele kleine Geldauflagen ausgesprochen, die sich in ihrer Höhe, neben dem vorliegenden Delikt, oft auch an den finanziellen Möglichkeiten der Betroffenen orientieren. Ratenzahlungsvereinbarungen sind nicht selten.

\section{Ein uneinheitlicher Markt}

Immer wieder wird davon gesprochen, dass Richter Geldauflagen aussprechen. Dreiviertel aller Gelder werden aber von Staats- und Amtsanwälten ausgesprochen. Aber auch Finanzämter für Fahndungen und Strafsachen, Zollämter, die Arbeitsagenturen oder Wohngeldämter greifen zu diesem Mittel. In einigen Bundesländern gibt es sogenannte Gnadenstellen, die Geldauflagen mit den Betroffenen vereinbaren. In über eintausend Behörden können mehr als zehntausend Mitarbeitende (Juristen) Geldauflagen aussprechen. So arbeiten in NordrheinWestfalen circa 2.500 potenzielle " $\mathrm{Zu}$ weiser « und in Schleswig-Holstein nur 300. In Hamburg gibt es einen Sammelfonds, der zweimal im Jahr Geldaufla- 
gen nach Vorlage umfassender Anträge an Einrichtungen vergibt, die sich in Hamburg engagieren. In Berlin gibt es für kleine, lokal tätige Einrichtungen einen Fonds, für den auf nur einer Website im Internet ein Antrag bis zu einer Höhe von 5.000 Euro gestellt werden kann.

In diesem Spannungsfeld stehen die gemeinnützigen Organisationen, die nach Geldauflagen fragen. Und das mit ganz unterschiedlichem Erfolg. Besonders erfolgreich sind mit jeweils über eine Million Euro per anno der Weiße Ring, die Deutsche Krebshilfe und Ärzte ohne Grenzen.

Es gibt keine eindeutigen Antworten zu den Erfolgskriterien, die eine größere Bereitschaft zur Unterstützung durch die Justiz bewirken. Bestimmt sind Bekanntheit und Seriosität zwei wichtige Voraussetzungen. Eindeutiger ist die Unterstützung von Opferschutzverbänden, wie der Weiße Ring mit über 1,8 Millionen Euro in 2011 zu erklären, da sich solche Einrichtungen direkt mit den Auswirkungen von Strafrechtsdelikten auf die unmittelbar Geschädigten befassen. Dadurch ist eine enge Verbindung zu Strafrechtsverfahren der Richter und Staatsanwälte gegeben. Aber schon im Bereich von Umwelt- und Naturschutz fallen die Zuweisungsgrößen bei ähnlich aufgestellten Organisationen ganz unterschiedlich aus.

\section{Chance für kleine Vereine}

Aber noch ein anderer Trend ist zu erkennen: In den letzten Jahren werden von den örtlichen Amtsgerichten und Staatsanwaltschaften zunehmend mehr Geldauflagen an kleine, lokal oder regional tätige Einrichtungen, wie $\mathrm{Ge}$ sundheitszentren, Kindergärten oder Kultureinrichtungen verteilt. Das liegt auch an den zunehmenden Fundraisingaktivitäten der lokalen Einrichtungen, um ausreichende finanzielle Mittel für ihre Arbeit einwerben zu können.

Zugleich können regionale Organisationen einen Vorteil nutzen: Durch die räumliche Nähe zu den zuweisenden Behörden können sie ihre Arbeit vor Ort darstellen. Viele Zuweiser unterstützen Projekte, die in ihrem direkten Umfeld tätig sind. Mit geringem finanziellem Aufwand können gerade kleine Einrichtungen bei »ihren « Gerichten und Staatsanwaltschaften um Geldauflagen anfragen.
Am Anfang steht immer steht der Eintrag in die Listen der gemeinnützigen Einrichtungen als Empfänger von Geldauflagen in Ermittlungs-, Straf- und Gnadenverfahren. Oberlandesgerichte oder Generalstaatsanwaltschaften führen diese »Bußgeldlisten « zum Nachweis der Gemeinnützigkeit der Organisationen, die Geldauflagen einwerben. Aufgrund der föderalen Struktur der Bundesrepublik ist jeder Eintrag Sache eines Bundeslandes und fällt somit unterschiedlich aus. In der Regel alle zwei Jahre sollte dieser Eintrag wiederholt werden. Änderungen in der Vereinssatzung, ein neuer Freistellungsbescheid von der Körperschaftssteuer oder Veränderungen im Vorstand führen zu einer vorzeitigen Aktualisierung.

Besonders lokal arbeitende Organisationen sollten sich bemühen, sich in den Listen der örtlichen Amts- und Landgerichte sowie der Staatsanwaltschaften eintragen zu lassen.

\section{Viel hilft nicht viel!}

Alles in allem stellt sich der "Zuweisermarkt" heute uneinheitlicher dar. Richter und Staatsanwälte reagieren unterschiedlich auf die Ansprache durch die Organisationen. Was bei der einen Einrichtung akzeptiert wird, führt bei der anderen zu Kritik.

In der Regel haben die Gemeinnützigen nur die Möglichkeit, die Zuweiser mit Mailings auf sich aufmerksam zu machen. Bei der Menge an Post, die jeden Tag auf den Schreibtischen der Richter und Staatsanwälte ankommt, kann dann schon mal der Hinweis aus der Justiz kommen, bitte zukünftig von der Zusendung abzusehen oder nur ein Schreiben an den Behördenleiter zu senden.

Die Erfahrung zeigt, dass für jede Organisation das Maß an Ansprachen unterschiedlich ausfällt und dass es wichtig ist, diesen Rhythmus zu erkennen. Denn auch hier gilt: Wer nicht fragt, bekommt auch nichts. Dabei hilft viel nicht viel. Richter und Staatsanwälte sind mit der Bewältigung ihrer originären Arbeit stark belastet. Da ist die Zusendung von Jahresberichten und dicken Broschüren nicht sinnvoll. Kurz und knapp ist hier gefragt. Die potenziellen Zuweiser müssen die Möglichkeit haben, schnell einen vorgestellten Sachverhalt und die angeboten Lösungsansätze zu erkennen.
Denn nur dadurch wird die Notwendigkeit zur Unterstützung der gemeinnützigen Arbeit deutlich. Die Bereitschaft zu Hilfe steigt nicht mit der Papiermenge oder mit der Informationsdichte!

Ein weiterer Faktor für eine erfolgreiche Ansprache ist, die potenziellen Zuweiser auch wirklich zu erreichen. Neben einigen Justizbehörden, die erklärtermaßen nur nichtpersonalisierte Anfragen annehmen, ist das klassische personalisierte Anschreiben eine wichtige Voraussetzung für eine erfolgreiche Unterstützung mit Geldauflagen. Dabei muss jede Organisation für sich entscheiden, ob die Zuweiser nach unterschiedlichen Segmentierungen angeschrieben werden. Dafür ist nicht nur das finanzielle Budget ein Maßstab. Beispiel: Eine Hochschule, die aus allen Teilen Deutschlands Studenten aufnimmt, kann in bestimmten Bundesländern keine Geldauflagen einwerben, weil sie dort vor Ort nicht tätig ist. Anders verhält es sich mit Entwicklungsorganisationen, die bundesweit um die Zuweisung von Geldern erfolgreich anfragen.

Fazit: Geldauflagen sind heute ein Bestandteil zur Finanzierung gemeinnütziger Arbeit. Jede bundesweit tätige Organisation, jeder lokal agierende Verein muss sich eine Position im "Zuweisermarkt« erarbeiten. Eine Überfrachtung mit $\mathrm{zu}$ vielen Informationen ist kontraproduktiv und verringert die Unterstützungsbereitschaft durch Richter und Staatsanwälte. Diese Zielgruppen möglichst "punktgenau « anzusprechen, ist eine wichtige Voraussetzung für den Erfolg. 